\title{
Complete Weight Enumerators of a Family of Three-Weight Linear Codes
}

\author{
Shudi Yang · Zheng-An Yao
}

Received: date / Accepted: date

\begin{abstract}
Linear codes have been an interesting topic in both theory and practice for many years. In this paper, for an odd prime $p$, we present the explicit complete weight enumerator of a family of $p$-ary linear codes constructed with defining set. The weight enumerator is an immediate result of the complete weight enumerator, which shows that the codes proposed in this paper are three-weight linear codes. Additionally, all nonzero codewords are minimal and thus they are suitable for secret sharing.
\end{abstract}

Keywords Linear code - Complete weight enumerator - Gaussian period . Gauss sum

Mathematics Subject Classification (2010) 94B15 · $11 \mathrm{~T} 71$

\section{Introduction}

Throughout this paper, let $p$ be an odd prime and $r=p^{m}$ for a positive integer $m \geq 2$. Denote by $\mathbb{F}_{r}$ a finite field with $r$ elements. An $[n, \kappa, \delta]$ linear code $C$ over $\mathbb{F}_{p}$ is a $\kappa$-dimensional subspace of $\mathbb{F}_{p}^{n}$ with minimum distance $\delta$ [7,28].

Let $A_{i}$ denote the number of codewords with Hamming weight $i$ in a linear code $C$ of length $n$. The (ordinary) weight enumerator of $C$ is defined by

$$
A_{0}+A_{1} z+A_{2} z^{2}+\cdots+A_{n} z^{n},
$$

S.D. Yang

Department of Mathematics, Sun Yat-sen University, Guangzhou 510275 and School of Mathematical Sciences, Qufu Normal University, Shandong 273165, P.R.China

Tel.: +86-15602338023

E-mail: yangshd3@mail2.sysu.edu.cn

Z.-A. Yao

Department of Mathematics, Sun Yat-sen University, Guangzhou 510275, P.R. China

E-mail: mcsyao@mail.sysu.edu.cn 
where $A_{0}=1$. The sequence $\left(A_{0}, A_{1}, A_{2}, \cdots, A_{n}\right)$ is called the (ordinary) weight distribution of the code $C$.

The complete weight enumerator of a code $C$ over $\mathbb{F}_{p}$ enumerates the codewords according to the number of symbols of each kind contained in each codeword. Denote elements of the field by $\mathbb{F}_{p}=\left\{w_{0}, w_{1}, \cdots, w_{p-1}\right\}$, where $w_{0}=0$. Also let $\mathbb{F}_{p}^{*}$ denote $\mathbb{F}_{p} \backslash\{0\}$. For a codeword $\mathrm{c}=\left(c_{0}, c_{1}, \cdots, c_{n-1}\right) \in \mathbb{F}_{p}^{n}$, let $w[\mathrm{c}]$ be the complete weight enumerator of $\mathrm{c}$, which is defined as

$$
w[\mathbf{c}]=w_{0}^{k_{0}} w_{1}^{k_{1}} \cdots w_{p-1}^{k_{p-1}},
$$

where $k_{j}$ is the number of components of c equal to $w_{j}, \sum_{j=0}^{p-1} k_{j}=n$. The complete weight enumerator of the code $C$ is then

$$
\operatorname{CWE}(C)=\sum_{\mathrm{c} \in C} w[\mathrm{c}] .
$$

The weight distribution of a linear code has attracted a lot of interests for many years and we refer the reader to $10,12,16,17,18,24,27,30,31,32$, 34, 35, 36, 37] and references therein for an overview of the related researches. It is not difficult to see that the complete weight enumerators are just the (ordinary) weight enumerators for binary linear codes. While for nonbinary linear codes, the weight enumerators can be obtained from their complete weight enumerators.

The information of the complete weight enumerator of a linear code is of vital use both in theory and in practice. For instance, Blake and Kith investigated the complete weight enumerator of Reed-Solomon codes and showed that they could be helpful in soft decision decoding 3, 20. In [19, the study of the monomial and quadratic bent functions was related to the complete weight enumerators of linear codes. It was illustrated by Ding et al. 9,11] that complete weight enumerators can be applied to calculate the deception probabilities of certain authentication codes. In 5, 6, 13, the authors studied the complete weight enumerators of some constant composition codes and presented some families of optimal constant composition codes.

However, it is extremely difficult to evaluate the complete weight enumerators of linear codes in general and there is little information on this topic in literature besides the above mentioned $3,5,6,13,20$. Kuzmin and Nechaev investigated the generalized Kerdock code and related linear codes over Galois rings and determined their complete weight enumerators in 21] and 22. Very recently, the authors in 25] obtained the complete weight enumerators of some cyclic codes by using Gauss sums. Li et al. 23] treated some linear codes by using exponential sums and Galois theory. In this paper, we shall determine the complete weight enumerators of a class of linear codes over finite fields.

Set $\bar{D}=\left\{d_{1}, d_{2}, \cdots, d_{n}\right\} \subseteq \mathbb{F}_{r}$. Denote by $\operatorname{Tr}$ the trace function from $\mathbb{F}_{r}$ to $\mathbb{F}_{p}$. A linear code associated with $\bar{D}$ is defined by

$$
C_{\bar{D}}=\left\{\left(\operatorname{Tr}\left(a d_{1}\right), \operatorname{Tr}\left(a d_{2}\right), \cdots, \operatorname{Tr}\left(a d_{n}\right)\right): a \in \mathbb{F}_{r}\right\} .
$$

Then $\bar{D}$ is called the defining set of this code $C_{\bar{D}}$ (see [8,14,15] for details). 
It should be noted that the authors in [8, 14] and [15] gave the definitions of the code $C_{\bar{D}}$ and the defining set $\bar{D}$. The authors in [14] established binary linear codes $C_{\bar{D}}$ with three weights. In [8], Ding proposed the general construction of the linear codes and determined their weights especially for three specific codes. The authors in [15] presented the defining set $\bar{D}=\left\{x \in \mathbb{F}_{r}^{*}: \operatorname{Tr}\left(x^{2}\right)=0\right\}$ to construct a class of linear codes $C_{\bar{D}}$ with two or three weights and investigated their applications in secret sharing, and furthermore, their complete weight enumerators were established by Yang and Yao 33.

In this paper, let $r=p^{m}$. The defining set $D$ is given by

$$
D=\left\{x \in \mathbb{F}_{r}^{*}: \operatorname{Tr}(x)=0\right\},
$$

and let

$$
C_{D}=\left\{\left(\operatorname{Tr}\left(a x^{2}\right)\right)_{x \in D}: a \in \mathbb{F}_{r}\right\} .
$$

For this kind of linear codes, we will study their complete weight enumerators, and their weight enumerators as well. We should mention that the main idea of this paper indeed comes from 8, 15. As will be proved later, they are linear codes with three weights. In addition, each nonzero codeword of $C_{D}$ constructed in this paper is minimal if $m \geq 4$, which indicates that the linear codes can be of use in secret sharing schemes [4] with nice access structures. The reader is referred to 15 for more information about this application.

The main results of this paper are given below.

Theorem 1 Let $N_{a}(\rho)=\#\left\{x \in \mathbb{F}_{r}^{*}: \operatorname{Tr}(x)=0\right.$ and $\left.\operatorname{Tr}\left(a x^{2}\right)=\rho\right\}$ with $\rho \in \mathbb{F}_{p}$ and $a \in \mathbb{F}_{r}$. Then the code $C_{D}$ of (2) has parameters $\left[p^{m-1}-1, m\right]$ and its complete weight enumerator is given in Table 1 if $m$ is even and Table 2 if $m$ is odd.

Table 1 Complete weight enumerator of the code $C_{D}$ if $m$ is even

\begin{tabular}{lll}
\hline$N_{a}(0)$ & $N_{a}(\rho)(\rho \neq 0)$ & Frequency \\
\hline$p^{m-2}-1$ & $p^{m-2}+\bar{\eta}(\rho) p^{\frac{m-2}{2}}$ & $\frac{p^{m}-p^{m-1}}{2}$ \\
$p^{m-2}-1$ & $p^{m-2}-\bar{\eta}(\rho) p^{\frac{m-2}{2}}$ & $\frac{p^{m}-p^{m-1}}{2}$ \\
$p^{m-2}-1-(p-1) p^{\frac{m-2}{2}}$ & $p^{m-2}+p^{\frac{m-2}{2}}$ & $\frac{1}{2}\left(p^{\frac{m}{2}}+1\right)\left(p^{\frac{m-2}{2}}-1\right)$ \\
$p^{m-2}-1+(p-1) p^{\frac{m-2}{2}}$ & $p^{m-2}-p^{\frac{m-2}{2}}$ & $\frac{1}{2}\left(p^{\frac{m}{2}}-1\right)\left(p^{\frac{m-2}{2}}+1\right)$ \\
$p^{m-1}-1$ & 0 & 1 \\
\hline
\end{tabular}

where $\bar{\eta}$ denotes the quadratic character of $\mathbb{F}_{p}$.

Theorem 2 The weight distribution of the code $C_{D}$ of (2) is given in Table 3 if $m$ is even and Table 4 if $m$ is odd.

Example 1 (i) Let $(p, m)=(3,4)$. Then by Theorems 1 and 2, the code $C_{D}$ has parameters $[26,4,12]$ with complete weight enumerator

$$
w_{0}^{26}+16 w_{0}^{14} w_{1}^{6} w_{2}^{6}+27 w_{0}^{8} w_{1}^{6} w_{2}^{12}+27 w_{0}^{8} w_{1}^{12} w_{2}^{6}+10 w_{0}^{2} w_{1}^{12} w_{2}^{12},
$$


Table 2 Complete weight enumerator of the code $C_{D}$ if $m$ is odd

\begin{tabular}{lll}
\hline$N_{a}(0)$ & $N_{a}(\rho)(\rho \neq 0)$ & Frequency \\
\hline$p^{m-2}-1$ & $p^{m-2}+\bar{\eta}(\rho) p^{\frac{m-1}{2}}$ & $\frac{p^{m-1}-1}{2}$ \\
$p^{m-2}-1$ & $p^{m-2}-\bar{\eta}(\rho) p^{\frac{m-1}{2}}$ & $\frac{p^{m-1}-1}{2}$ \\
$p^{m-2}+(p-1) p^{\frac{m-3}{2}}$ & $p^{m-2}-p^{\frac{m-3}{2}}$ & $\frac{p-1}{2}\left(p^{m-1}+p^{\frac{m-1}{2}}\right)$ \\
$p^{m-2}-(p-1) p^{\frac{m-3}{2}}$ & $p^{m-2}+p^{\frac{m-3}{2}}$ & $\frac{p-1}{2}\left(p^{m-1}-p^{\frac{m-1}{2}}\right)$ \\
$p^{m-1}-1$ & 0 & 1 \\
\hline
\end{tabular}

where $\bar{\eta}$ denotes the quadratic character of $\mathbb{F}_{p}$.

Table 3 The weight distribution of $C_{D}$ if $m$ is even

\begin{tabular}{ll}
\hline Weight $i$ & Frequency $A_{i}$ \\
\hline$(p-1) p^{m-2}$ & $p^{m}-p^{m-1}$ \\
$(p-1)\left(p^{m-2}+p^{\frac{m-2}{2}}\right)$ & $\frac{1}{2}\left(p^{\frac{m}{2}}+1\right)\left(p^{\frac{m-2}{2}}-1\right)$ \\
$(p-1)\left(p^{m-2}-p^{\frac{m-2}{2}}\right)$ & $\frac{1}{2}\left(p^{\frac{m}{2}}-1\right)\left(p^{\frac{m-2}{2}}+1\right)$ \\
0 & 1 \\
\hline
\end{tabular}

Table 4 The weight distribution of $C_{D}$ if $m$ is odd

\begin{tabular}{ll}
\hline Weight $i$ & Frequency $A_{i}$ \\
\hline$(p-1) p^{m-2}$ & $p^{m-1}-1$ \\
$(p-1)\left(p^{m-2}-p^{\frac{m-3}{2}}\right)$ & $\frac{p-1}{2}\left(p^{m-1}+p^{\frac{m-1}{2}}\right)$ \\
$(p-1)\left(p^{m-2}+p^{\frac{m-3}{2}}\right)$ & $\frac{p-1}{2}\left(p^{m-1}-p^{\frac{m-1}{2}}\right)$ \\
0 & 1 \\
\hline
\end{tabular}

and weight enumerator

$$
1+16 z^{12}+54 z^{18}+10 z^{24} .
$$

These results coincide with numerical computation by Magma.

(ii) Let $(p, m)=(5,3)$. Then by Theorems 1 and 2 , the code $C_{D}$ has parameters $[24,3,16]$ with complete weight enumerator

$$
w_{0}^{24}+60 w_{0}^{8} w_{1}^{4} w_{2}^{4} w_{3}^{4} w_{4}^{4}+12 w_{0}^{4} w_{1}^{10} w_{4}^{10}+12 w_{0}^{4} w_{2}^{10} w_{3}^{10}+40 w_{1}^{6} w_{2}^{6} w_{3}^{6} w_{4}^{6} .
$$

and weight enumerator

$$
1+60 z^{16}+24 z^{20}+40 z^{24}
$$

These results are confirmed by Magma.

The remainder of this paper is organized as follows. Section 2 recalls some definitions and results on Gaussian periods and Gauss sums over finite fields. Section 3 is devoted to the proof of Theorem 11. Section 4 shows that every nonzero codeword of the code is minimal. Section 5 concludes this paper. 


\section{Mathematical foundations}

We start with cyclotomic classes and Gaussian periods over finite fields. Recall that $r=p^{m}$. Let $\alpha$ be a fixed primitive element of $\mathbb{F}_{r}$ and $r-1=s N$, where $s, N$ are two positive integers with $s>1$ and $N>1$. Define $C_{i}^{(N, r)}=\alpha^{i}\left\langle\alpha^{N}\right\rangle$ for $i=0,1, \cdots, N-1$, where $\left\langle\alpha^{N}\right\rangle$ denotes the subgroup of $\mathbb{F}_{r}^{*}$ generated by $\alpha^{N}$. The cosets $C_{i}^{(N, r)}$ are called the cyclotomic classes of order $N$ in $\mathbb{F}_{r}$. Obviously, $\# C_{i}^{(N, r)}=\frac{r-1}{N}$.

Set $\zeta_{p}=\exp \left(\frac{2 \pi \sqrt{-1}}{p}\right)$. The Gaussian periods of order $N$ are defined by

$$
\eta_{i}^{(N, r)}=\sum_{x \in C_{i}^{(N, r)}} \chi_{1}(x) .
$$

Here $\chi_{1}(x)=\zeta_{p}^{\operatorname{Tr}(x)}$ is the canonical additive character of $\mathbb{F}_{r}$, where $\operatorname{Tr}$ is the trace function from $\mathbb{F}_{r}$ to $\mathbb{F}_{p}$ defined by

$$
\operatorname{Tr}(x)=\sum_{i=0}^{m-1} x^{p^{i}}, \quad x \in \mathbb{F}_{r} .
$$

The orthogonal property of canonical additive character, which can be easily checked, is given by

$$
\sum_{x \in \mathbb{F}_{r}} \chi_{1}(a x)= \begin{cases}r & \text { if } a=0 \\ 0 & \text { if } a \in \mathbb{F}_{r}^{*}\end{cases}
$$

Some results on Gaussian periods are given below [29].

Lemma 1 [29] Let $r=p^{m}$. When $N=2$, the Gaussian periods are given by

$$
\eta_{0}^{(2, r)}=\left\{\begin{array}{lll}
\frac{-1+(-1)^{m-1} \sqrt{r}}{2} & \text { if } p \equiv 1 \quad(\bmod 4), \\
\frac{-1+(-1)^{m-1}(\sqrt{-1})^{m} \sqrt{r}}{2} & \text { if } p \equiv 3 \quad(\bmod 4),
\end{array}\right.
$$

and $\eta_{1}^{(2, r)}=-1-\eta_{0}^{(2, r)}$.

For later use, we introduce Gauss sums in the following. Let $\eta$ be the quadratic character of $\mathbb{F}_{r}\left[15\right.$. The quadratic Gauss sum $G\left(\eta, \chi_{1}\right)$ over $\mathbb{F}_{r}$ is defined by

$$
G\left(\eta, \chi_{1}\right)=\sum_{x \in \mathbb{F}_{r}^{*}} \eta(x) \chi_{1}(x)=\sum_{x \in \mathbb{F}_{r}} \eta(x) \chi_{1}(x),
$$

and the quadratic Gauss sum $G\left(\bar{\eta}, \bar{\chi}_{1}\right)$ over $\mathbb{F}_{p}$ is defined by

$$
G\left(\bar{\eta}, \bar{\chi}_{1}\right)=\sum_{x \in \mathbb{F}_{p}^{*}} \bar{\eta}(x) \bar{\chi}_{1}(x)=\sum_{x \in \mathbb{F}_{p}} \bar{\eta}(x) \bar{\chi}_{1}(x),
$$

where $\bar{\eta}$ and $\bar{\chi}_{1}$ are the quadratic character and canonical additive character of $\mathbb{F}_{p}$, respectively.

The following lemmas will be needed in the sequel. 
Lemma 2 (See Theorems 5.15 [26]) With the symbols and notation above, we have

$$
G\left(\eta, \chi_{1}\right)=(-1)^{m-1}(\sqrt{-1})^{\frac{(p-1)^{2}}{4} m} \sqrt{r}
$$

where $r=p^{m}$, and

$$
G\left(\bar{\eta}, \bar{\chi}_{1}\right)=(\sqrt{-1})^{\frac{(p-1)^{2}}{4}} \sqrt{p} .
$$

Lemma 3 (See Theorem 5.33 of [26]) Let $r=p^{m}$ and $f(x)=a_{2} x^{2}+a_{1} x+$ $a_{0} \in \mathbb{F}_{r}[x]$ with $a_{2} \neq 0$. Then

$$
\sum_{x \in \mathbb{F}_{r}} \chi_{1}(f(x))=\chi_{1}\left(a_{0}-a_{1}^{2}\left(4 a_{2}\right)^{-1}\right) \eta\left(a_{2}\right) G\left(\eta, \chi_{1}\right)
$$

Lemma 4 (See Lemma 7 of [15]) If $m \geq 2$ is even, then $\eta(y)=1$ for each $y \in \mathbb{F}_{p}^{*}$. If $m$ is odd, then $\eta(y)=\bar{\eta}(y)$ for each $y \in \mathbb{F}_{p}$.

Lemma 5 (15, 25] $)$ For each $c \in \mathbb{F}_{p}$, let $t_{c}=\#\left\{a \in \mathbb{F}_{r}: \operatorname{Tr}\left(a^{2}\right)=c\right\}$. Then

$$
t_{c}= \begin{cases}p^{m-1} & \text { if } m \text { odd and } c=0, \\ p^{m-1}+\bar{\eta}(c)(-1)^{\frac{p-1}{2} \frac{m-1}{2}} p^{\frac{m-1}{2}} & \text { if } m \text { odd and } c \neq 0, \\ p^{m-1}-(-1)^{\frac{p-1}{2} \frac{m}{2}}(p-1) p^{\frac{m-2}{2}} & \text { if } m \text { even and } c=0, \\ p^{m-1}+(-1)^{\frac{p-1}{2} \frac{m}{2}} p^{\frac{m-2}{2}} & \text { if } m \text { even and } c \neq 0 .\end{cases}
$$

\section{The proofs of the main results}

Let notation be as before. Our task of this section is to prove Theorem 1 depicted in Section 1, while Theorem 2 follows immediately from Theorem 1 . Below we present some auxiliary results before proving the main results of this paper.

Lemma 6 Let $a \in \mathbb{F}_{r}^{*}$ and $\rho \in \mathbb{F}_{p}^{*}$. Then we have

$$
\begin{aligned}
& \sum_{z \in \mathbb{F}_{p}^{*}} \sum_{y \in \mathbb{F}_{p}} \sum_{x \in \mathbb{F}_{r}} \zeta_{p}^{\operatorname{Tr}\left(a z x^{2}+y x\right)-z \rho} \\
& = \begin{cases}(-1)^{\frac{p-1}{2} \frac{m}{2}} \eta(a) p^{\frac{m+2}{2}} & \text { if } m \text { even and } \operatorname{Tr}\left(a^{-1}\right)=0, \\
-(-1)^{\frac{p-1}{2} \frac{m+2}{2}} \eta(a) \bar{\eta}\left(\operatorname{Tr}\left(a^{-1}\right)\right) \bar{\eta}(\rho) p^{\frac{m+2}{2}} & \text { if } m \text { even and } \operatorname{Tr}\left(a^{-1}\right) \neq 0, \\
(-1)^{\frac{p-1}{2} \frac{m-1}{2}} \eta(a) \bar{\eta}(\rho) p^{\frac{m+3}{2}} & \text { if } m \text { odd and } \operatorname{Tr}\left(a^{-1}\right)=0, \\
-(-1)^{\frac{p-1}{2} \frac{m-1}{2}} \eta(a) \bar{\eta}\left(\operatorname{Tr}\left(a^{-1}\right)\right) p^{\frac{m+1}{2}} & \text { if } m \text { odd and } \operatorname{Tr}\left(a^{-1}\right) \neq 0 .\end{cases}
\end{aligned}
$$


Proof It follows from Lemmas 3 and 4 that

$$
\begin{aligned}
& \sum_{z \in \mathbb{F}_{p}^{*}} \sum_{y \in \mathbb{F}_{p}} \sum_{x \in \mathbb{F}_{r}} \zeta_{p}^{\operatorname{Tr}\left(a z x^{2}+y x\right)-z \rho} \\
& =\sum_{z \in \mathbb{F}_{p}^{*}} \zeta_{p}^{-z \rho} \sum_{y \in \mathbb{F}_{p}} \sum_{x \in \mathbb{F}_{r}} \zeta_{p}^{\operatorname{Tr}\left(a z x^{2}+y x\right)} \\
& =\sum_{z \in \mathbb{F}_{p}^{*}} \zeta_{p}^{-z \rho} \sum_{y \in \mathbb{F}_{p}} \chi_{1}\left(-\frac{y^{2}}{4 a z}\right) \eta(a z) G\left(\eta, \chi_{1}\right) \\
& =\eta(a) G\left(\eta, \chi_{1}\right) \sum_{z \in \mathbb{F}_{p}^{*}} \zeta_{p}^{-z \rho} \eta(z) \sum_{y \in \mathbb{F}_{p}} \zeta_{p}^{-\frac{1}{4 z} \operatorname{Tr}\left(a^{-1}\right) y^{2}} \\
& = \begin{cases}p \eta(a) G\left(\eta, \chi_{1}\right) \sum_{z \in \mathbb{F}_{p}^{*}} \zeta_{p}^{-z \rho} \eta(z) & \text { if } \operatorname{Tr}\left(a^{-1}\right)=0 \\
\eta(a) G\left(\eta, \chi_{1}\right) \sum_{z \in \mathbb{F}_{p}^{*}} \zeta_{p}^{-z \rho} \eta(z) \bar{\eta}\left(-\frac{\operatorname{Tr}\left(a^{-1}\right)}{4 z}\right) G\left(\bar{\eta}, \bar{\chi}_{1}\right) \text { if } \operatorname{Tr}\left(a^{-1}\right) \neq 0\end{cases} \\
& = \begin{cases}p \eta(a) G\left(\eta, \chi_{1}\right) \sum_{z \in \mathbb{F}_{p}^{*}} \zeta_{p}^{-z \rho} \eta(z) & \text { if } m \text { even and } \operatorname{Tr}\left(a^{-1}\right)=0, \\
\eta(a) \bar{\eta}\left(-\operatorname{Tr}\left(a^{-1}\right)\right) G\left(\eta, \chi_{1}\right) G\left(\bar{\eta}, \bar{\chi}_{1}\right) \sum_{z \in \mathbb{F}_{p}^{*}} \zeta_{p}^{-z \rho} \eta(z) \bar{\eta}(z) \text { if } \operatorname{Tr}\left(a^{-1}\right) \neq 0\end{cases} \\
& = \begin{cases}-p \eta(a) G\left(\eta, \chi_{1}\right) & \text { if } m \text { odd and } \operatorname{Tr}\left(a^{-1}\right)=0, \\
\eta(a) \bar{\eta}\left(-\operatorname{Tr}\left(a^{-1}\right)\right) \bar{\eta}(-\rho) G\left(\eta, \chi_{1}\right) G\left(\bar{\eta}, \bar{\chi}_{1}\right)^{2} & \text { if } m \text { even and } \operatorname{Tr}\left(a^{-1}\right) \neq 0, \\
p \eta(a) \bar{\eta}(-\rho) G\left(\eta, \chi_{1}\right) G\left(\bar{\eta}, \bar{\chi}_{1}\right) & \text { if } m, \operatorname{Tr}\left(a^{-1}\right) \neq 0 . \\
-\eta(a) \bar{\eta}\left(-\operatorname{Tr}\left(a^{-1}\right)\right) G\left(\eta, \chi_{1}\right) G\left(\bar{\eta}, \bar{\chi}_{1}\right)\end{cases}
\end{aligned}
$$

From Lemma 2, we get the desired conclusions.

Lemma 7 For any $a \in \mathbb{F}_{r}^{*}$ and any $\rho \in \mathbb{F}_{p}^{*}$, let

$$
N_{a}(\rho)=\#\left\{x \in \mathbb{F}_{r}^{*}: \operatorname{Tr}(x)=0 \text { and } \operatorname{Tr}\left(a x^{2}\right)=\rho\right\} .
$$

Then we have

$$
N_{a}(\rho)=\left\{\begin{array}{c}
p^{m-2}+(-1)^{\frac{p-1}{2} \frac{m}{2}} \eta(a) p^{\frac{m-2}{2}} \\
\text { if } m \text { even and } \operatorname{Tr}\left(a^{-1}\right)=0, \\
p^{m-2}-(-1)^{\frac{p-1}{2} \frac{m+2}{2}} \eta(a) \bar{\eta}\left(\operatorname{Tr}\left(a^{-1}\right)\right) \bar{\eta}(\rho) p^{\frac{m-2}{2}} \\
\text { if } m \text { even and } \operatorname{Tr}\left(a^{-1}\right) \neq 0, \\
p^{m-2}+(-1)^{\frac{p-1}{2} \frac{m-1}{2}} \eta(a) \bar{\eta}(\rho) p^{\frac{m-1}{2}} \\
\text { if } m \text { odd and } \operatorname{Tr}\left(a^{-1}\right)=0, \\
p^{m-2}-(-1)^{\frac{p-1}{2} \frac{m-1}{2}} \eta(a) \bar{\eta}\left(\operatorname{Tr}\left(a^{-1}\right)\right) p^{\frac{m-3}{2}} \\
\text { if } m \text { odd and } \operatorname{Tr}\left(a^{-1}\right) \neq 0 .
\end{array}\right.
$$

Proof We point out that

$$
N_{a}(\rho)=\#\left\{x \in \mathbb{F}_{r}: \operatorname{Tr}(x)=0 \text { and } \operatorname{Tr}\left(a x^{2}\right)=\rho\right\}
$$


since $\rho \neq 0$. Hence, for any $a \in \mathbb{F}_{r}^{*}$ and any $\rho \in \mathbb{F}_{p}^{*}$, we have

$$
\begin{aligned}
N_{a}(\rho) & =p^{-2} \sum_{x \in \mathbb{F}_{r}}\left(\sum_{y \in \mathbb{F}_{p}} \zeta_{p}^{y \operatorname{Tr}(x)}\right)\left(\sum_{z \in \mathbb{F}_{p}} \zeta_{p}^{z\left(\operatorname{Tr}\left(a x^{2}\right)-\rho\right)}\right) \\
& =p^{-2} \sum_{y \in \mathbb{F}_{p}^{*}} \sum_{x \in \mathbb{F}_{r}} \zeta_{p}^{\operatorname{Tr}(y x)}+p^{-2} \sum_{z \in \mathbb{F}_{p}^{*}} \sum_{y \in \mathbb{F}_{p}} \sum_{x \in \mathbb{F}_{r}} \zeta_{p}^{\operatorname{Tr}\left(a z x^{2}+y x\right)-z \rho}+p^{m-2} .
\end{aligned}
$$

Note that

$$
\sum_{y \in \mathbb{F}_{p}^{*}} \sum_{x \in \mathbb{F}_{r}} \zeta_{p}^{\operatorname{Tr}(y x)}=0
$$

since

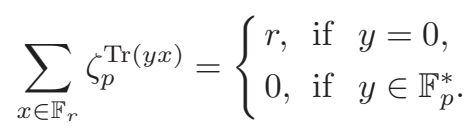

The desired conclusions then follow from Lemma 6 ,

In order to calculate the frequency of the complete weight enumerator of codewords in $C_{D}$, we shall compute

$$
\#\left\{a \in \mathbb{F}_{r}^{*}: \eta(a)= \pm 1 \text { and } \operatorname{Tr}\left(a^{-1}\right)=0\right\}
$$

and

$$
\#\left\{a \in \mathbb{F}_{r}^{*}: \eta(a) \bar{\eta}\left(\operatorname{Tr}\left(a^{-1}\right)\right)= \pm 1\right\},
$$

which are given in the following three lemmas.

Lemma 8 For any $a \in \mathbb{F}_{r}^{*}$, let

$$
n_{i}=\#\left\{a \in \mathbb{F}_{r}^{*}: \eta(a)=i \text { and } \operatorname{Tr}\left(a^{-1}\right)=0\right\}, \quad i \in\{1,-1\} .
$$

Then, for $m$ being odd, we have

$$
n_{1}=n_{-1}=\frac{p^{m-1}-1}{2},
$$

and for $m$ being even, we have

$$
\left\{\begin{array}{l}
n_{1}=\frac{r-1}{2 p}+\frac{p-1}{p} \eta_{0}^{(2, r)} \\
n_{-1}=\frac{r-1}{2 p}+\frac{p-1}{p} \eta_{1}^{(2, r)}
\end{array}\right.
$$

where $\eta_{0}^{(2, r)}$ and $\eta_{1}^{(2, r)}$ are given in Lemma 1 . 
Proof Observe that $n_{-1}=n-n_{1}=p^{m-1}-1-n_{1}$. Thus, we only focus on $n_{1}$. Let $\alpha$ be a fixed primitive element of $\mathbb{F}_{r}$. Then $\mathbb{F}_{r}^{*}=\langle\alpha\rangle$ and $\mathbb{F}_{p}^{*}=\left\langle\alpha^{\frac{r-1}{p-1}}\right\rangle$.

Note that $\eta(a)=1$ if and only if $a \in C_{0}^{(2, r)}$.

For the case of $m$ being even, we have $\mathbb{F}_{p}^{*} \subseteq C_{0}^{(2, r)}$ since 2 divides $\frac{r-1}{p-1}$.

Therefore, we obtain

$$
\begin{aligned}
n_{1} & =\sum_{x \in C_{0}^{(2, r)}} \frac{1}{p} \sum_{y \in \mathbb{F}_{p}} \zeta_{p}^{y \operatorname{Tr}(x)} \\
& =\frac{r-1}{2 p}+\frac{1}{p} \sum_{y \in \mathbb{F}_{p}^{*}} \sum_{x \in C_{0}^{(2, r)}} \zeta_{p}^{\operatorname{Tr}(y x)} \\
& =\frac{r-1}{2 p}+\frac{p-1}{p} \eta_{0}^{(2, r)}
\end{aligned}
$$

where $\eta_{0}^{(2, r)}$ is given in Lemma 1 .

Similarly, for the case of $m$ being odd, we have $\frac{r-1}{p-1} \equiv 1 \bmod 2$, from which the asserted result follows.

Lemma 9 For any $a \in \mathbb{F}_{r}^{*}$, let

$$
n_{i, j}=\#\left\{a \in \mathbb{F}_{r}^{*}: \eta(a)=i \text { and } \bar{\eta}\left(\operatorname{Tr}\left(a^{-1}\right)\right)=j\right\}, \quad i, j \in\{1,-1\} .
$$

Then, for $m$ being even, we have

$$
n_{1,1}=n_{1,-1}=\frac{p-1}{4}\left(p^{m-1}+(-1)^{\frac{p-1}{2} \frac{m}{2}} p^{\frac{m-2}{2}}\right)
$$

and for $m$ being odd, we have

$$
\left\{\begin{array}{l}
n_{1,1}=\frac{p-1}{4}\left(p^{m-1}+(-1)^{\frac{p-1}{2} \frac{m-1}{2}} p^{\frac{m-1}{2}}\right), \\
n_{1,-1}=\frac{p-1}{4}\left(p^{m-1}-(-1)^{\frac{p-1}{2} \frac{m-1}{2}} p^{\frac{m-1}{2}}\right) .
\end{array}\right.
$$

Proof We note the following fact:

$$
\begin{aligned}
n_{1, j} & =\#\left\{a \in \mathbb{F}_{r}^{*}: a \in C_{0}^{(2, r)}, \bar{\eta}\left(\operatorname{Tr}\left(a^{-1}\right)\right)=j\right\} \\
& =\frac{1}{2} \#\left\{a \in \mathbb{F}_{r}^{*}: \bar{\eta}\left(\operatorname{Tr}\left(a^{2}\right)\right)=j\right\}, \quad j \in\{1,-1\} .
\end{aligned}
$$

Then, the desired results follow from Lemma 5 .

Lemma 10 For any $a \in \mathbb{F}_{r}^{*}$, let

$$
s_{i}=\#\left\{a \in \mathbb{F}_{r}^{*}: \eta(a) \bar{\eta}\left(\operatorname{Tr}\left(a^{-1}\right)\right)=i\right\}, \quad i \in\{1,-1\} .
$$

Then, for $m$ being even, we have

$$
s_{1}=s_{-1}=\frac{p^{m}-p^{m-1}}{2},
$$


and for $m$ being odd, we have

$$
\left\{\begin{array}{l}
s_{1}=\frac{p-1}{2}\left(p^{m-1}+(-1)^{\frac{p-1}{2} \frac{m-1}{2}} p^{\frac{m-1}{2}}\right), \\
s_{-1}=\frac{p-1}{2}\left(p^{m-1}-(-1)^{\frac{p-1}{2} \frac{m-1}{2}} p^{\frac{m-1}{2}}\right) .
\end{array}\right.
$$

Proof Since $\bar{\eta}$ and Tr are balanced, we have

$$
\begin{aligned}
& \#\left\{a \in \mathbb{F}_{r}^{*}: \bar{\eta}\left(\operatorname{Tr}\left(a^{-1}\right)\right)=-1\right\} \\
& =\frac{1}{2} \#\left\{a \in \mathbb{F}_{r}^{*}: \operatorname{Tr}\left(a^{-1}\right) \neq 0\right\} \\
& =\frac{p^{m}-p^{m-1}}{2} .
\end{aligned}
$$

Following from the definition above, we deduce that

$$
\begin{aligned}
s_{1} & =\#\left\{a \in \mathbb{F}_{r}^{*}: \eta(a) \bar{\eta}\left(\operatorname{Tr}\left(a^{-1}\right)\right)=1\right\} \\
& =n_{1,1}+n_{-1,-1} \\
& =n_{1,1}+\#\left\{a \in \mathbb{F}_{r}^{*}: \bar{\eta}\left(\operatorname{Tr}\left(a^{-1}\right)\right)=-1\right\}-n_{1,-1} \\
& =n_{1,1}-n_{1,-1}+\frac{p^{m}-p^{m-1}}{2} .
\end{aligned}
$$

Similarly,

$$
\begin{aligned}
s_{-1} & =\#\left\{a \in \mathbb{F}_{r}^{*}: \eta(a) \bar{\eta}\left(\operatorname{Tr}\left(a^{-1}\right)\right)=-1\right\} \\
& =n_{1,-1}-n_{1,1}+\frac{p^{m}-p^{m-1}}{2} .
\end{aligned}
$$

The asserted results then follow from Lemma 9

Remark 1 Lemma 10 determines $s_{1}$ and $s_{-1}$ with the help of Lemma 9. In the following, we give another short proof for the case of $m$ being even.

If $m$ is even, Lemma 4 states that $\eta(y)=1$ for each $y \in \mathbb{F}_{p}^{*}$. And there exists a $y^{\prime} \in \mathbb{F}_{p}^{*}$ such that $\bar{\eta}\left(y^{\prime}\right)=-1$. So $\eta\left(y^{\prime}\right) \bar{\eta}\left(y^{\prime}\right)=-1$.

It follows that

$$
\begin{aligned}
s_{1} & =\#\left\{a \in \mathbb{F}_{r}^{*}: \eta(a) \bar{\eta}\left(\operatorname{Tr}\left(a^{-1}\right)\right)=1\right\} \\
& =\#\left\{a \in \mathbb{F}_{r}^{*}: \eta(a) \bar{\eta}(\operatorname{Tr}(a))=1\right\} \\
& =\#\left\{y^{\prime} a \in \mathbb{F}_{r}^{*}: \eta\left(y^{\prime} a\right) \bar{\eta}\left(\operatorname{Tr}\left(y^{\prime} a\right)\right)=1\right\} \\
& =\#\left\{y^{\prime} a \in \mathbb{F}_{r}^{*}: \eta\left(y^{\prime}\right) \bar{\eta}\left(y^{\prime}\right) \eta(a) \bar{\eta}(\operatorname{Tr}(a))=1\right\} \\
& =\#\left\{a \in \mathbb{F}_{r}^{*}: \eta(a) \bar{\eta}(\operatorname{Tr}(a))=-1\right\}=s_{-1} .
\end{aligned}
$$

Hence we have $s_{1}=s_{-1}=\frac{p^{m}-p^{m-1}}{2}$ since

$$
s_{1}+s_{-1}=\#\left\{a \in \mathbb{F}_{r}^{*}: \operatorname{Tr}\left(a^{-1}\right) \neq 0\right\}=p^{m}-p^{m-1} .
$$


3.1 The proof of Theorem 1

It is now sufficient to show the complete weight enumerator of $C_{D}$ as stated in Theorem 1. Recall that

$$
C_{D}=\left\{\left(\operatorname{Tr}\left(a x^{2}\right)\right)_{x \in D}: a \in \mathbb{F}_{r}\right\},
$$

where $D=\left\{x \in \mathbb{F}_{r}^{*}: \operatorname{Tr}(x)=0\right\}$.

It is obvious that the code $C_{D}$ has length $n=p^{m-1}-1$ and dimension $m$.

Observe that $a=0$ gives the zero codeword and the contribution to the complete weight enumerator is $w_{0}^{n}$. Hence, we assume that $a \in \mathbb{F}_{r}^{*}$ for the rest of the proof.

For each codeword $\left(\operatorname{Tr}\left(a x^{2}\right)\right)_{x \in D}$ of $C_{D}$ and $\rho \in \mathbb{F}_{p}$, we should consider the number of solutions $x \in \mathbb{F}_{r}^{*}$ satisfying $\operatorname{Tr}(x)=0$ and $\operatorname{Tr}\left(a x^{2}\right)=\rho$, i.e.,

$$
N_{a}(\rho)=\#\left\{x \in \mathbb{F}_{r}^{*}: \operatorname{Tr}(x)=0 \text { and } \operatorname{Tr}\left(a x^{2}\right)=\rho\right\} .
$$

Note that $N_{a}(0)=p^{m-1}-1-\sum_{\rho \in \mathbb{F}_{p}^{*}} N_{a}(\rho)$. Thus we only need to calculate $N_{a}(\rho)$ with $\rho \in \mathbb{F}_{p}^{*}$, which is shown in Lemma 7.

The desired conclusions of Theorem 1 then follow from Lemmas 7 , 8 and 10.

Note that for even $m$ and $\rho \in \mathbb{F}_{p}^{*}$, the frequencies of the codewords with $N_{a}(\rho)=p^{m-2}+(-1)^{\frac{p-1}{2} \frac{m}{2}} p^{\frac{m-2}{2}}$ and $N_{a}(\rho)=p^{m-2}-(-1)^{\frac{p-1}{2} \frac{m}{2}} p^{\frac{m-2}{2}}$ are $\frac{p^{m}-1}{2 p}+\frac{p-1}{p} \eta_{0}^{(2, r)}$ and $\frac{p^{m}-1}{2 p}+\frac{p-1}{p} \eta_{1}^{(2, r)}$, respectively. The results are given in Table 1 according to Lemma 1.

\section{Minimal codewords in $C_{D}$}

In this section, we will show that each codeword of $C_{D}$ given by (2) is minimal. Hence, we need to introduce some definitions [15].

The support of a vector $\mathbf{c}=\left(c_{0}, \cdots, c_{n-1}\right) \in \mathbb{F}_{p}^{n}$ is defined as

$$
\left\{0 \leq i \leq n-1: c_{i} \neq 0\right\} \text {. }
$$

We say that a vector $\mathbf{x}$ covers a vector $\mathbf{y}$ if the support of $\mathbf{x}$ contains that of $\mathbf{y}$ as a proper subset.

A minimal codeword of a linear code $C$ is a nonzero codeword that does not cover any other nonzero codeword of $C$.

If each nonzero codeword of $C$ is minimal, then the secret sharing scheme based on the dual code $C^{\perp}$ may have nice access structure, see Theorem 12 of 15 . However, it is still very hard to construct such a linear code $C$. We list the following lemma for minimal codewords [1,2].

Lemma 11 Every nonzero codeword of a linear code $C$ over $\mathbb{F}_{p}$ is minimal, provided that

$$
\frac{w_{\min }}{w_{\max }}>\frac{p-1}{p},
$$

where $w_{\min }$ and $w_{\max }$ denote the minimum and maximum nonzero weights in $C$, respectively. 
For the linear codes $C_{D}$ of (2), if $m$ is even and $m \geq 4$, we have

$$
\frac{w_{\min }}{w_{\max }}=\frac{p^{m-2}-p^{\frac{m-2}{2}}}{p^{m-2}+p^{\frac{m-2}{2}}}>\frac{p-1}{p} .
$$

The case of $m \geq 4$ being odd is proved similarly, see also 15 .

Using Lemma 11, we conclude that all the nonzero codewords of $C_{D}$ are minimal if $m \geq 4$. Therefore, we can construct secret sharing schemes based on the dual codes $C_{\bar{D}}^{\perp}$ with nice access structures. We omit the details here since it is similar to that of [15].

\section{Concluding remarks}

Inspired by [8, 15], we constructed a family of three-weight linear codes. Their complete weight enumerators and weight enumerators were presented explicitly in this paper. We also showed that every nonzero codeword of $C_{D}$ is minimal and thus the dual codes $C_{D}^{\perp}$ can be applied to construct secret sharing schemes.

Acknowledgements The work of Zheng-An Yao is partially supported by the NSFC (Grant No.11271381), the NSFC (Grant No.11431015) and China 973 Program (Grant No. 2011CB808000). This work is also partially supported by the NSFC (Grant No. 61472457) and Guangdong Natural Science Foundation (Grant No. 2014A030313161).

\section{References}

1. Ashikhmin A., Barg A.: Minimal vectors in linear codes. IEEE Transactions on Information Theory 44(5), 2010-2017 (1998)

2. Ashikhmin A., Barg A., Cohen G., Huguet L.: Variations on minimal codewords in linear codes. In: Applied Algebra, Algebraic Algorithms and Error-Correcting Codes, Lecture Notes in Computer Science, vol. 948, pp. 96-105. Springer Berlin Heidelberg (1995)

3. Blake I.F., Kith K.: On the complete weight enumerator of Reed-Solomon codes. SIAM J. Discret. Math. 4(2), 164-171 (1991)

4. Carlet C., Ding C., Yuan J.: Linear codes from perfect nonlinear mappings and their secret sharing schemes. IEEE Transactions on Information Theory 51(6), 2089-2102 (2005)

5. Chu W., Colbourn C.J., Dukes P.: On constant composition codes. Discrete Applied Mathematics 154(6), 912-929 (2006)

6. Ding C.: Optimal constant composition codes from zero-difference balanced functions. IEEE Transactions on Information Theory 54(12), 5766-5770 (2008)

7. Ding C.: Codes from Difference Sets. Singapore: World Scientific (2015)

8. Ding C.: Linear codes from some 2-designs. IEEE Transactions on Information Theory 61(6), 3265-3275 (2015)

9. Ding C., Helleseth, T., Klove, T., Wang, X.: A generic construction of Cartesian authentication codes. IEEE Transactions on Information Theory 53(6), 2229-2235 (2007)

10. Ding C., Liu Y., Ma C., Zeng L.: The weight distributions of the duals of cyclic codes with two zeros. IEEE Transactions on Information Theory 57(12), 8000-8006 (2011)

11. Ding C., Wang X.: A coding theory construction of new systematic authentication codes. Theoretical Computer Science 330(1), 81-99 (2005) 
12. Ding C., Yang J.: Hamming weights in irreducible cyclic codes. Discrete Mathematics 313(4), 434-446 (2013)

13. Ding C., Yin J.: A construction of optimal constant composition codes. Designs, Codes and Cryptography 40(2), 157-165 (2006)

14. Ding K., Ding C.: Binary linear codes with three weights. IEEE Communications Letters 18(11), 1879-1882 (2014)

15. Ding K., Ding C.: A class of two-weight and three-weight codes and their applications in secret sharing. IEEE Transactions on Information Theory 61(11), 5835-5842 (2015)

16. Dinh H.Q., Li C., Yue Q.: Recent progress on weight distributions of cyclic codes over finite fields. Journal of Algebra Combinatorics Discrete Structures and Applications 2(1), 39-63 (2015)

17. Feng K., Luo J.: Weight distribution of some reducible cyclic codes. Finite Fields and Their Applications 14(2), 390-409 (2008)

18. Feng T.: On cyclic codes of length $2^{2^{r}}-1$ with two zeros whose dual codes have three weights. Designs, Codes and Cryptography 62(3), 253-258 (2012)

19. Helleseth T., Kholosha A.: Monomial and quadratic bent functions over the finite fields of odd characteristic. IEEE Transactions on Information Theory 52(5), 2018-2032 (2006)

20. Kith K.: Complete weight enumeration of Reed-Solomon codes. Master's thesis, Department of Electrical and Computing Engineering, University of Waterloo, Waterloo, Ontario, Canada (1989)

21. Kuzmin A., Nechaev A.: Complete weight enumerators of generalized Kerdock code and linear recursive codes over Galois ring. In: Workshop on coding and cryptography, pp. 333-336 (1999)

22. Kuzmin A., Nechaev A.: Complete weight enumerators of generalized Kerdock code and related linear codes over Galois ring. Discrete Applied Mathematics 111(1), 117-137 (2001)

23. Li C., Bae S., Ahn J., Yang S., Yao Z.: Complete weight enumerators of some linear codes and their applications. Designs, Codes and Cryptography (2015). DOI 10.1007/ s10623-015-0136-9

24. Li C., Yue Q.: Weight distributions of two classes of cyclic codes with respect to two distinct order elements. IEEE Transactions on Information Theory 60(1), 296-303 (2014)

25. Li C., Yue Q., Fu F.: Complete weight enumerators of some cyclic codes. Designs, Codes and Cryptography (2015). DOI 10.1007/s10623-015-0091-5

26. Lidl R., Niederreiter, H.: Finite Fields. Addison-Wesley, Reading, MA (1983)

27. Luo J., Feng K.: On the weight distributions of two classes of cyclic codes. IEEE Transactions on Information Theory 54(12), 5332-5344 (2008)

28. MacWilliams F.J., Sloane N.J.A.: The Theory of Error-Correcting Codes. NorthHolland Publishing, Amsterdam (1977)

29. Myerson G.: Period polynomials and Gauss sums for finite fields. Acta Arithmetica 39(3), 251-264 (1981)

30. Sharma A., Bakshi G.K.: The weight distribution of some irreducible cyclic codes. Finite Fields and Their Applications 18(1), 144-159 (2012)

31. Vega G.: The weight distribution of an extended class of reducible cyclic codes. IEEE Transactions on Information Theory 58(7), 4862-4869 (2012)

32. Wang B., Tang C., Qi Y., Yang Y., Xu M.: The weight distributions of cyclic codes and elliptic curves. IEEE Transactions on Information Theory 58(12), 7253-7259 (2012)

33. Yang S., Yao Z.: Complete weight enumerators of some linear codes (2015). arXiv:1505.06326

34. Yu L., Liu H.: The weight distribution of a family of $p$-ary cyclic codes. Designs, Codes and Cryptography (2014). DOI 10.1007/s10623-014-0029-3

35. Yuan J., Carlet C., Ding C.: The weight distribution of a class of linear codes from perfect nonlinear functions. IEEE Transactions on Information Theory 52(2), 712-717 (2006)

36. Zheng D., Wang X., Zeng X., Hu L.: The weight distribution of a family of $p$-ary cyclic codes. Designs, Codes and Cryptography 75(2), 263-275 (2015)

37. Zhou, Z., Ding, C.: A class of three-weight cyclic codes. Finite Fields and Their Applications 25, 79-93 (2014) 